Cerebral venous thrombosis may mimic extraaxial hemorrages on unenhanced head $\mathrm{CT}$, and hemorrhagic venous infarctions may be misinterpreted as parenchymal contusion, leading to an incorrect diagnosis of primary traumatic brain injury in infants. (Krasnokutsky MV. Am J Roentgenol 2011;197(3):W503-7).

\title{
ENCEPHALOPATHIES
}

\section{EARLY HYPOTHERMIA AND OUTCOME OF ACUTE ENCEPHALOPATHY AND ENCEPHALITIS}

Outcome of acute encephalopathy/encephalitis was compared in children treated with early or delayed cooling in a study at St Mary's Hospital, Fukuoka, and other centers in Japan. Children between 1 month and 14 years old, cared for at pediatric intensive care units at 10 Japanese tertiary centers, Jan 1997-July 2008, were retrospectively enrolled. Eight centers provided therapeutic hypothermia within $48 \mathrm{~h}$ of diagnosis. Of 43 children, 27 had a preceding viral infection, and 17 were influenza. Acute necrotizing encephalopathy was diagnosed in 8 children, hemorrhagic shock and encephalopathy syndrome in 5, and acute encephalopathy with refractory seizures in 16 . The incidence of unfavorable outcome for children cooled after $12 \mathrm{~h}$ of diagnosis was invariant with normothermic children but was significantly higher compared with children cooled within $12 \mathrm{~h}$. Younger age $<18$ months and marked elevation of serum lactic dehydrogenase (LD) greater than the $75^{\text {th }}$ percentile were associated with severe disability or death after 12 months. Neurologic outcome evaluated using Pediatric Cerebral Performance Scale at 12 months was dependent on the CT findings of cerebral edema, serum LD levels and Glasgow Coma Scale at admission. (Kawano G, Iwata O, Iwata S, et al. Determinants of outcomes following acute child encephalopathy and encephalitis: pivotal effect of early and delayed cooling. Arch Dis Child October 2011;96:936-941). (Respond: Osuke Iwata, Department of Paediatrics, Kurume University School of Medicine, 67 Asahmachi, Kurume, Fukuoka, 830-0011 Japan. Email: o.iwata@orbix.uk.net).

COMMENT. Early cooling in children with acute encephalopathy/encephalitis may be neuroprotective, whereas delayed cooling after $12 \mathrm{~h}$ of initial neurologic signs is likely to be deleterious.

Whole-body hypothermia for newborns with HIE. Researchers in Australia, New Zealand, Canada, and US determined the effectiveness and safety of moderate wholebody hypothermia (33.5C for 72 hours) commenced within 6 hours of birth in 110 term and near-term newborns with HIE. Compared to standard care in 111 infants, infants treated with hypothermia had a reduced risk of death or major sensorineural disability at 2 years of age: $51.4 \%$ infants in the hypothermia group and $66.3 \%$ in the control group died or were disabled $(\mathrm{p}=0.03)$. Mortality rate decreased and survival rate without disability increased. (Jacobs SE, Morley CJ, Inder TE, et al. Arch Pediatr Adolesc Med 2011;165(8):692-700).

(Respond: Susan E Jacobs MD. E-mail: suejacobs@thewomens.org.au). 